\title{
Collective Rayleigh scattering from dielectric particles
}

\author{
S Mark Wiggins, Gordon R M Robb, Brian W J McNeil, \\ Dino A Jaroszynski, David R Jones and Steven P Jamison \\ University of Strathclyde, Department of Physics and Applied Physics, Glasgow G4 0NG, \\ UK \\ E-mail: g.r.m.robb@strath.ac.uk
}

Received 6 August 2001, accepted for publication 16 January 2002

Published 8 February 2002

Online at stacks.iop.org/MST/13/263

\begin{abstract}
An investigation is taking place into a new classical scattering phenomenon called 'collective Rayleigh scattering'. A collection of dielectric particles pumped by a laser radiation field may form a strong density grating on the scale of the radiation wavelength. The particles then coherently scatter the incident radiation. Current theoretical research is confined to collective Rayleigh scattering from particles small compared with the radiation wavelength, for which there are many possible applications in the field of nonlinear optics. However, by considering larger Mie particles, it can be seen that there are also potential applications in the areas of optical particle characterization and discrimination. This paper outlines the theoretical framework of CRS and the first observations from preliminary experiments utilizing a standing-wave gradient force trap.
\end{abstract}

Keywords: Rayleigh scattering, collective scattering, small dielectric particles, density grating, Mie scattering, optical trapping, gradient force trap

\section{Introduction}

Dielectric particles that are small compared to the wavelength of an incident radiation field exhibit the well known Rayleigh scattering behaviour [1]. A new classical scattering phenomenon called 'collective Rayleigh scattering' (CRS) [2] has been predicted which involves the spontaneous formation of a particle density modulation on the scale of the radiation wavelength, thereby forming a refractive density grating. Radiation scattering from such an ensemble of particles is therefore coherent. In the field of non-linear optics, these density gratings represent potentially novel nonlinear optical media with applications as artificial Kerr media [3], tunable photonic bandgap materials [4] and also to facilitate feedback in random lasers [5]. A potentially useful application in the field of optical particle characterization is outlined in this paper.

The grating may be spontaneously generated via the interaction of the particles with a radiation pump field and a small counterpropagating radiation probe field (which may arise from noise due to random fluctuations in the particle density) that produces periodic ponderomotive forces in the particle ensemble. The collective nature of CRS results in an exponentially growing counter-propagating radiation field. The phenomenon is analogous to the periodic bunching of free electrons in the free-electron laser (FEL) [6] and atoms in the collective atomic recoil laser (CARL) [7], both processes resulting in the emission of coherent radiation.

Besides the theoretical framework of CRS, the practical considerations required to experimentally observe this phenomenon in an optical trap are also presented here. The initial experimental set-up is that of a standing-wave gradient trap. Such a trap immediately imposes large periodic ponderomotive forces on the particles, bypassing the need for them to grow spontaneously. This configuration is being used to determine the feasibility of optically confining, for a long enough time frame, the large number of particles necessary to allow spontaneous CRS to occur. It will be emphasised that CRS is rapidly evolving process therefore this time frame can be short (of the order of ms or $\mu$ s). Steady-state trapping of large numbers of particles is not necessary. 


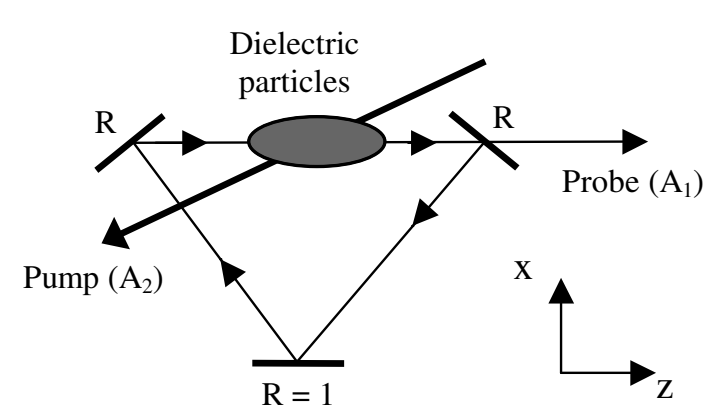

Figure 1. Schematic of a CRS experiment.

\section{Theory}

A simple 1D model is used to demonstrate the principle of CRS. It consists of a strong plane pump wave, scattered by an initially uniform spatial distribution of dielectric Rayleigh particles, and an initially very weak plane wave probe field. The probe field is approximately counterpropagating to the pump field (the pump-probe misalignment is exaggerated in the figure for clarity), and is enclosed in a unidirectional ring cavity, as shown schematically in figure 1. The medium is taken to be free space.

The form of the $E$-field in the cavity is

$$
E=E_{1}(z, t)+E_{2}(z, t)
$$

where $E_{1}(z, t)=\left[A_{1}(t) \mathrm{e}^{\mathrm{i} k(z-c t)}+\right.$ c.c. $] \hat{x}$ is the $E$-field of the initially weak probe field and $E_{2}(z, t)=\left[A_{2} \mathrm{e}^{-\mathrm{i} k(z+c t)}+\right.$ c.c. $] \hat{x}$ is the $E$-field of the strong pump field, which we assume here to be of constant amplitude, $c$ is the speed of light in vacuo, $k=2 \pi / \lambda$ is the wavenumber and $\hat{x}$ is a transverse unit vector. It is assumed that only one mode of the cavity undergoes significant interaction with the particles and that both the pump and probe fields are resonant with a cavity mode, i.e. $k=l \pi / L_{\text {cav }}$ where $l$ is an integer and $L_{\text {cav }}$ is the cavity roundtrip length.

The force on the $j$ th particle can be derived from the Lorentz force equation to be [2]

$$
F_{j}=\frac{\partial d_{j}}{\partial t} \times B\left(z_{j}, t\right)
$$

where $B(z, t)=\frac{1}{c} \hat{z} \times\left(E_{1}-E_{2}\right)$ is the magnetic field due to the electromagnetic waves in the cavity, $d_{j}$ is the dipole moment of the $j$ th particle induced by the $E$-field at $z=z_{j}$ the axial position of the $j$ th particle given by

$$
d_{j}=\varepsilon_{0} V_{p}\left[\chi\left(A_{1}(t) \mathrm{e}^{\mathrm{i} k(z-c t)}+A_{2} \mathrm{e}^{-\mathrm{i} k(z+c t)}\right)+\text { c.c. }\right] \hat{x}
$$

$\varepsilon_{0}$ is the permittivity of free space and $\chi=\chi_{1}+\chi_{2}$ is the susceptibility, $V_{p}=4 \pi a^{3} / 3$ is the volume and $a$ is the radius, respectively, of the particle. Substituting for $B$ and $d_{j}$ in equation (2) and following the derivation described in [2], the dynamics of the particles under the influence of the electromagnetic fields are described by

$$
\begin{gathered}
\frac{\mathrm{d} z_{j}}{\mathrm{~d} t}=\frac{p_{j}}{M} \\
\frac{\mathrm{d} p_{j}}{\mathrm{~d} t}=2 \varepsilon_{0} V_{p}\left[\mathrm{i} \chi A_{2}\left(A_{1}(t) \mathrm{e}^{2 \mathrm{i} k z_{j}}-\text { c.c. }\right)+\chi_{2}\left(\left|A_{1}(t)\right|^{2}-A_{2}^{2}\right)\right]
\end{gathered}
$$

where $p_{j}$ is the $z$-component of the momentum of the $j$ th particle and $M$ is the mass of the particle. The dynamics of the probe field are found from Maxwell's wave equation

$$
\left(\frac{\partial^{2}}{\partial z^{2}}-\frac{1}{c^{2}} \frac{\partial^{2}}{\partial t^{2}}\right) E(z, t)=\mu_{0} \frac{\partial^{2} P(z, t)}{\partial t^{2}}
$$

where $\mu_{0}$ is the permeability of free space and $P(z, t)$ is a macroscopic polarisation arising from the contributions of the dipole moments of all the particles, i.e.

$$
P(z, t)=\sum_{j=1}^{N} d_{j} \delta\left(r-r_{j}(t)\right) .
$$

Substituting for $E$ and $d_{j}$ and again following the derivation of [2], it can be shown that the evolution of the complex amplitude of the probe field, $A_{1}(t)$, is described by

$$
\frac{\mathrm{d} A_{1}}{\mathrm{~d} t}=\frac{\mathrm{i} k V_{p} A_{2} \chi N_{p}}{2}\left\langle\mathrm{e}^{\mathrm{i} \theta}\right\rangle-\frac{c T}{L_{c a v}} A_{1}
$$

where $\langle\cdots\rangle=\frac{1}{N} \sum_{j=1}^{N}(\cdots)_{j}$ denotes an average over the ensemble of $N$ particles in the interaction region, $N_{p}$ is the average number density of particles in the cavity interaction volume. Radiation losses at the mirrors are modelled using a phenomenological loss of $T A_{1}$ per cavity round trip, i.e. a loss rate of $c T A_{1} / L_{c a v}$ where $T=1-R$ is the mirror transmission coefficient and $R$ is the corresponding reflection coefficient.

As shown in [2], equations (4), (5) and (8) can be reduced to a universally scaled form with a minimum number of free parameters in the limit $\chi_{1} \gg \chi_{2}$, where the dispersive part of the susceptibility dominates the absorptive part:

$$
\begin{gathered}
\frac{\mathrm{d} \theta_{j}}{\mathrm{~d} \bar{t}}=\bar{p}_{j} \\
\frac{\mathrm{d} \bar{p}_{j}}{\mathrm{~d} \bar{t}}=-\left(\bar{A}_{1} \mathrm{e}^{\mathrm{i} \theta_{j}}+\text { c.c. }\right) \\
\frac{\mathrm{d} \bar{A}_{1}}{\mathrm{~d} \bar{t}}=\left\langle\mathrm{e}^{-\mathrm{i} \theta}\right\rangle-\kappa \bar{A}_{1}
\end{gathered}
$$

using the dimensionless parameters given by

$$
\begin{gathered}
\theta_{j}=2 k z_{j}, \quad \bar{p}_{j}=\frac{p_{j}}{\rho_{c} M c^{2}}, \quad \bar{t}=\frac{4 \pi \rho_{c} c t}{\lambda} \\
\bar{A}_{1}=-2 \mathrm{i} \sqrt{\frac{\varepsilon_{0}}{\rho_{c} N_{p} M c^{2}}} A_{1}, \quad \kappa=\frac{\lambda}{4 \pi \rho_{c}} \frac{T}{L_{c a v}} \\
\rho_{c}=\left(\frac{\varepsilon_{0} \chi_{1}^{2} A_{2}^{2} N_{p} V_{p}^{2}}{4 M c^{2}}\right)^{1 / 3}
\end{gathered}
$$

where $\kappa$ is a cavity loss parameter and $\rho_{c}$ is a gain parameter. The initial conditions for equations (9) correspond to a very weak probe field intensity and particles with a uniform distribution of positions and a Gaussian momentum distribution with width $\sigma_{p}$ given by

$$
\sigma_{p}=\frac{1}{\rho_{c}} \sqrt{\frac{k_{B} T}{M c^{2}}}
$$

where $k_{B}$ is Boltzmann's constant and $T$ is the temperature. 


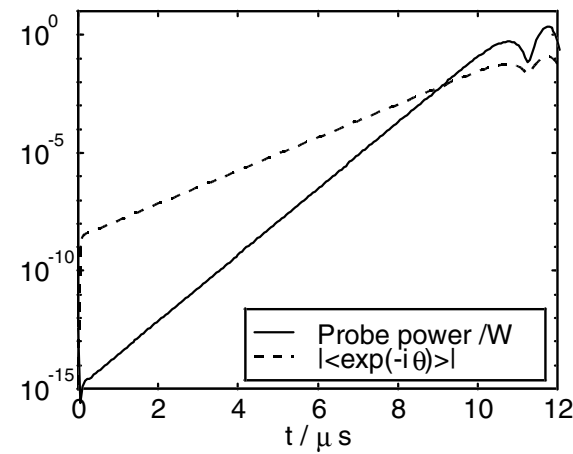

Figure 2. CRS simulation showing an exponential increase in the probe power as a function of time.

\section{Example simulation}

As an example of CRS, we consider a collection of free latex nanoparticles $(a=25 \mathrm{~nm}$, refractive index $n=1.59$, density $=1.05 \times 10^{-3} \mathrm{~kg} \mathrm{~m}^{3}, \chi=\left(n^{2}-1\right) /\left(n^{2}+2\right)=0.34$ enclosed in a high-quality ring cavity. Other parameters used in the simulation are: pump power $P=1.25 \mathrm{~W}$, pump wavelength $\lambda_{0}=532 \mathrm{~nm}$, beam radius $=10 \mu \mathrm{m}, L_{\text {cav }}=$ $10 \mathrm{~cm}, R=0.96, N=4 \times 10^{6}$ and $T=300 \mathrm{~K}$.

Figure 2 shows the evolution of the probe field intensity and the bunching parameter $|\langle\exp (-\mathrm{i} \theta)\rangle|$ for the above parameters, calculated using equations (9). The bunching parameter represents the amplitude of the particle density modulation on the spatial scale $\lambda / 2$. Its value ranges from zero for uniformly distributed particles, to one for particles perfectly bunched at a single value of $\theta$. It can be seen from figure 2 that the simulation predicts very strong exponential amplification of the probe field simultaneous with the spontaneous formation of a strong particle density modulation on the radiation wavelength scale, the distinctive feature of CRS. Although the model at present contains several simplifying but restrictive assumptions (1D plane wave, constant pump field), the results it predicts are very interesting and clearly warrant further investigation. A more detailed model of CRS which should better represent a real experiment and includes, for example, pump depletion, pulse propagation, diffraction effects and Brownian motion, is currently being developed and will be described in a future publication. Initial results suggest that the principle features of CRS are still present when these additional effects are included in the model.

\section{Experimental requirements}

To observe CRS in an experiment, a large number of particles must be present within the scattering volume, i.e. the beam, for the time frame of the evolution. The planned experimental configuration resembles that of a single-beam gradient force trap or optical tweezers [8], which have been used for a number of years in the trapping and manipulation of small particles [9]. The pump beam is tightly focused to obtain a large intensity thereby maximizing the backscattered radiation probe field. A gas (air) has been selected as the background medium though a liquid medium may also be feasible. Gravitational settling of the sub-micron particles in the gas medium must be counteracted to some extent. The radial component of the

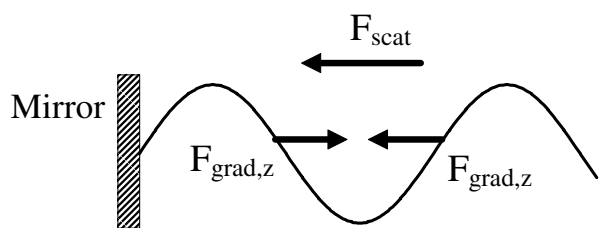

(a)

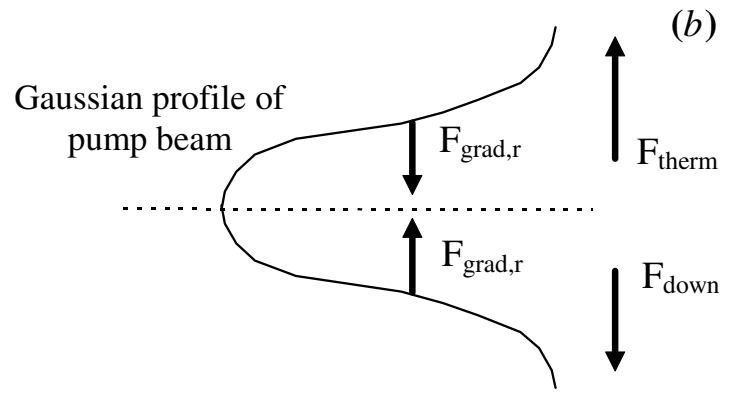

Figure 3. Forces acting $(a)$ axially and $(b)$ radially on a particle in the standing-wave gradient trap.

strong pump field has been proposed for this task such that the radial gradient force of the strongly focused pump beam, which is the time-averaged Lorentz force acting on the dipole induced by the pump field, is comparable to the downwards gravitational force.

Presented in this paper are the first results from preliminary experiments conducted with a standing-wave gradient force trap [10]. The pump beam is normally incident onto a mirror creating a standing wave of period $\lambda / 2$. With such a trap, the initial counterpropagating probe field (essentially the reflected pump field) is large so that strong ponderomotive forces are immediately imposed onto the particles. The particles should then quickly bunch at the anti-nodes of the standing wave, i.e. the particle density grating does not spontaneously build up from noise. It is evident that bunching should be easier to achieve with a standing-wave trap than with a single-beam trap.

The main motivation behind these experiments is to determine the number of particles that can be reasonably held within the scattering volume for a suitable time using an optical trap. For true CRS evolving spontaneously from noise, the number of particles should be sufficiently large so that the potential wells can each be occupied by a reasonable number of particles, enabling growth of the probe field to a value comparable with that of the pump.

A schematic representation of the forces on a particle in a gradient force trap is shown in figure 3. The gradient and scattering force may be, respectively, written in the form [11]

$$
\begin{aligned}
& F_{\text {grad }}(z, r)=\frac{2 \pi n_{2} a^{3}}{c}\left(\frac{m^{2}-1}{m^{2}+2}\right) \nabla I(z, r) \\
& F_{\text {scat }}(z)=\frac{8 \pi n_{2} k^{4} a^{6}}{3 c}\left(\frac{m^{2}-1}{m^{2}+2}\right)\langle S(z, r)\rangle_{T}
\end{aligned}
$$

where $n_{2}$ is the medium refractive index, $m=n / n_{2}$ is the relative refractive index of the particle, $k$ is the wavenumber, $\nabla I$ is the pump beam intensity gradient and $\langle S(z, r)\rangle_{T}$ is a time-averaged version of the Poynting vector. The formalism of [10] has been applied for the particular case of a standingwave trap. The steep intensity gradients of the standing wave 
define the axial gradient force, directed towards the anti-node in each period. If the beam waist is located at the mirror surface, then the scattering force is directed towards the mirror because of the unavoidable losses occurring upon reflection at the mirror surface. The radial gradient force, defined by the Gaussian profile of the pump beam cross section, is directed towards the beam axis. The downwards force (comprising gravity and buoyancy) is given by

$$
\left|F_{\text {down }}\right|=\frac{4 \pi a^{3} g}{3}\left(\rho-\rho_{2}\right)
$$

where $\rho$ and $\rho_{2}$ are the respective densities of the particle and medium.

A thermophoretic force arises from the absorption of pump energy by the mirror that sets up a convective flow in the medium [12]. It is directed upwards in the region of interest close to the mirror and its magnitude is given by

$$
\left|F_{\text {therm }}\right|=\frac{12 \pi \eta^{2} a|\nabla T|}{\rho_{2} T} \frac{C_{s}\left(\frac{\sigma_{2}}{\sigma}+C_{t} \frac{\lambda_{m f p}}{a}\right)}{\left(1+3 C_{m} \frac{\lambda_{m f p}}{a}\right)\left(1+2 \frac{\sigma_{2}}{\sigma}+2 C_{t} \frac{\lambda_{m f p}}{a}\right)}
$$

where $\eta, \sigma_{2}, T$ and $|\nabla T|$ are the medium viscosity, thermal conductivity, ambient temperature and temperature gradient respectively, $\sigma$ is the particle thermal conductivity, $C_{s}=1.17$, $C_{t}=2.18$ and $C_{m}=1.14$ are accommodation coefficients from gas kinetic theory and $\lambda_{m f p}$ is the mean free path of the medium molecules given by

$$
\lambda_{m f p}=\frac{k_{B} T}{4 \sqrt{2} \pi p a_{2}^{2}}
$$

where $p$ is the medium pressure and $a_{2}=0.185 \mathrm{~nm}$ is the molecule radius. At pressures close to atmospheric pressure, the convective flow can be so rapid as to dominate the process. Therefore, it is desirable to conduct experiments at a somewhat lower gas pressure to reduce the thermophoretic force (which is proportional to pressure), thus slowing the convective flow.

To hold a particle in a standing-wave trap four criteria must be met:

(a) The axial gradient force must exceed the scattering force for axial confinement.

(b) In the lower part of the beam, the sum of the upwards forces must exceed that of downwards forces to prevent particles falling out of the beam.

(c) In the upper part of the beam, the sum of the downwards forces must exceed that of the upwards forces to prevent particles being lifted up and out of the beam.

(d) The energetic depth of trap must greatly exceed the kinetic energy of the particle for trap stability, i.e. particle Brownian motion must be negligible.

Satisfying all criteria for many particles $\left(10^{2}-10^{6}\right)$ simultaneously would clearly be an extremely complicated to model and indeed difficult to obtain in experiment. However, CRS rapidly evolves on a $\mu \mathrm{s}-\mathrm{ms}$ time scale therefore true optical trapping of all particles is not a necessity. The purpose of the initial experiments is to optimize the experimental conditions such that the number of particles in the scattering volume for a given time interval is maximized.

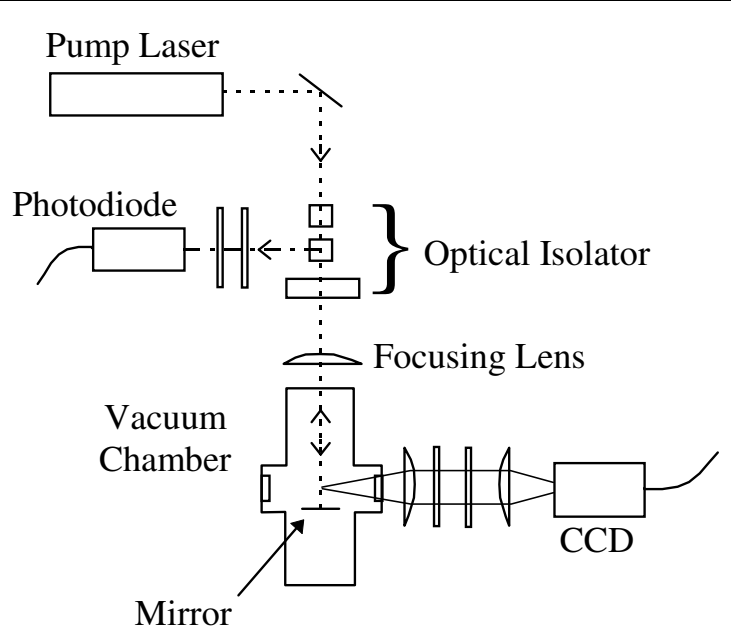

Figure 4. Schematic top view of the experimental set-up.

\section{Experimental set-up}

A top view of the experimental set-up with the standingwave trap is displayed in figure 4 . These experiments have been conducted at the University of Strathclyde TOPS user facility [13]. The pump laser is a $\mathrm{CW} \mathrm{Nd:} \mathrm{YVO}_{4}$ laser operating at wavelength $\lambda_{0}=532 \mathrm{~nm}$ and power $P$ up to $5 \mathrm{~W}$. It is focused onto the surface of a dielectric mirror inside the vacuum chamber. The particles are puffed into the evacuated chamber with a small amount of air and fall under gravity until they pass into the beam path, whereupon they are subject to the gradient forces. After initial particle injection the pressure is $\sim 150$ mbar. Through slow bleeding of the pressure, $\sim 10$ mbar can be achieved with many particles still present in the chamber. This allows for control of the thermophoretic force. Radiation scattered through the side viewports of the chamber allows the particles in the beam to be imaged with $\mathrm{CCD}$ and digital cameras.

The number of particles in the beam can be estimated from the measured intensity of the pump beam upon exiting the chamber. Assuming mono-sized particles and no multiple scattering, the attenuation of the pump field due to the presence of the particles is given by the Bouguer-Lambert-Beer (BLB) law

$$
I=I_{0} \exp \left[-N_{p} C_{e x t} h\right]
$$

where $I$ and $I_{0}$ are the final and initial beam intensities respectively, $N_{p}=2 N / \pi w_{0}^{2} h$ is the number density of the particles, the factor of 2 denotes the double crossing of the particles by the pump beam, $C_{\text {ext }}$ is the extinction cross section of the particles and $h$ is the distance traversed by the beam. Hence the number of particles in the beam $N$ is given by

$$
N \sim-\ln \left(\frac{I}{I_{0}}\right) \frac{\pi w_{0}^{2}}{4 C_{s c a}}
$$

assuming $C_{a b s} \cong 0$ and $C_{e x t} \cong C_{s c a}$ where $C_{a b s}$ and $C_{s c a}$ are the particle absorption and scattering cross sections respectively. The BLB law assumes no scattered light is detected, i.e. only the transmitted light is detected. However, when measuring the on-axis transmission, a real detector will also detect the forward scattered light in a finite solid 
angle about the axis. This diagnostic also picks up the backward scattered light in the same solid angle. To keep these contributions to the detected signal negligible $(<1 \%)$, the detector solid angle is $\sim 10$ mrad only.

Titanium dioxide $\left(\mathrm{TiO}_{2}\right)$ powder has been the particle source for these initial experiments. These particles have a large scattering cross section $(n=2.7)$ and a small absorption cross section $\left(\operatorname{Im}[n] \sim 10^{-4}\right)$ making them ideal for optical trapping. A particle size distribution analysis ${ }^{1}$ revealed a spread in particle radius from $100 \mathrm{~nm}$ to $2 \mu \mathrm{m}$. However, there were sufficient numbers of particles (around 1 in 7) in the desired range $(a=250 \mathrm{~nm} \pm 10 \%)$ as required by the experimental design. An initial wide size distribution is of no concern because of the self-selecting nature of the trapping process: the smallest particles have sufficient Brownian motion to escape the trap and the largest particles are subject to large scattering forces which expel them from the trap. These $\mathrm{TiO}_{2}$ particles do not satisfy the Rayleigh criterion $(a<\lambda / 10)$ and are so-called Mie particles $(a \sim \lambda)$ thus requiring the use of generalized Lorentz-Mie theory to model the scattering [14]. These particles would behave as Rayleigh particles with an infra-red pump beam $(\lambda>2.5 \mu \mathrm{m})$. Trapping them with a visible beam, however, remains a useful first experimental study because of the relative ease of operation and diagnosis in the visible region as compared with the infra-red. The gradient forces are also stronger at the shorter wavelength.

\section{Experimental observations}

Initial experiments with the pump beam focused to a waist size of $w_{0}=60 \mu \mathrm{m}$ and of power $P=2 \mathrm{~W}$ have demonstrated the influence of the thermophoretic force on the trapping process (the gas pressure here is $\sim 150$ mbar). Presented in figure 5 are photographs of particles in the pump beam at various time intervals up to $2 \mathrm{~h}$ after injection. Initially, particles are falling through the beam in the air currents used to inject them into the chamber, see figure 5(a). However, as these currents subside and the mirror heats up, the thermophoretic force increases in magnitude eventually dominating the evolution. This results in an upwards convective flow of particles through the beam, figures $5(b)-(d)$, i.e. there is no radial confinement of the particles. For the state depicted in figure $5(d)$, the average drop in power due to the presence of the particles is $\sim 16 \%$. Hence, from equation (7), at any one time the number of particles in the beam $N \sim 600$. The local temperature gradient is unknown therefore the thermophoretic force is estimated by equating it to the Stokes drag force $F_{\text {drag }}$ as the particle flows through the medium at a constant velocity, that is,

$$
\left|F_{\text {therm }}\right| \sim\left|F_{\text {drag }}\right|=\frac{6 \pi \eta a^{3} v}{C_{c}}
$$

where $v$ is the magnitude of the particle terminal velocity and $C_{c}$ is the Cunningham correction factor to Stokes' law due to the small particle size [15] given by

$$
C_{c} \cong 1+C_{1}\left[C_{2}+\exp \left(-C_{3} a / \lambda_{m f p}\right)\right] \frac{\lambda_{m f p}}{a}
$$

1 Measurements were made using a Horiba Instruments (Northampton, UK) LA-920 particle size analyser.

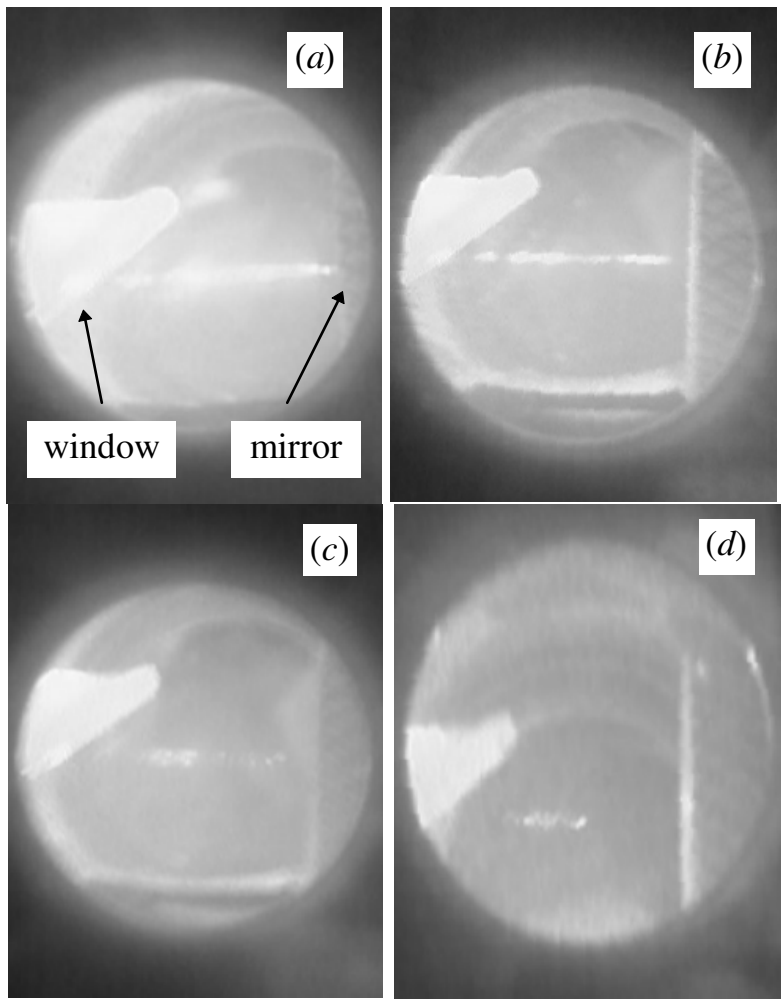

Figure 5. Photographs of particles in the pump beam at a time of (a) $t=30 \mathrm{~s},(b) 5 \mathrm{~min},(c) 30 \mathrm{~min}$ and (d) $180 \mathrm{~min}$ after particle injection into the vacuum chamber.

where $C_{1}=1.21, C_{2}=0.40$ and $C_{3}=0.78$ are empirical constants. The particle velocity is $\sim 3 \mathrm{~mm} \mathrm{~s}^{-1}$ hence $F_{\text {therm }} \sim$ $100 \mathrm{fN}$ which is much larger than $F_{\text {down }}=3 \mathrm{fN}$ or $F_{\text {grad }}(\max )$ $\sim 5 \mathrm{fN}$.

Focusing the pump beam more tightly to a waist of $30 \mu \mathrm{m}$ increases the radial gradient force to a maximum of $\sim 30 \mathrm{fN}$. Beam focusing is a compromise between achieving a large enough radial gradient force to hold the particles in the beam for the longest time and maintaining a long enough Rayleigh length $z_{R}=\pi w_{0} / \lambda$ to maximize the number of useful potential wells, i.e. length of the scattering volume. Reducing the gas pressure by a factor of 10 reduces the thermophoretic force to $\sim 10 \mathrm{fN}$. The radial gradient force is thus large enough to counteract the thermophoretic force and radial containment may occur. Indeed this has been observed experimentally. Longitudinally, however, with radially confined particles, rapid motion of the particles towards the mirror is observed. This is a consequence of Mie particles being used at this stage. The diameter of the $\mathrm{TiO}_{2}$ particles is almost equal to the pump wavelength so they each spatially occupy approximately two potential wells. The axial gradient forces exerted on these Mie particles are then effectively cancelled out leaving the scattering force directed towards the mirror as the dominant force.

\section{Application note}

When considering the larger particles with $a \leqslant \lambda$, it can be seen that there are potentially useful applications in the areas of optical particle characterization and discrimination. Rayleigh 


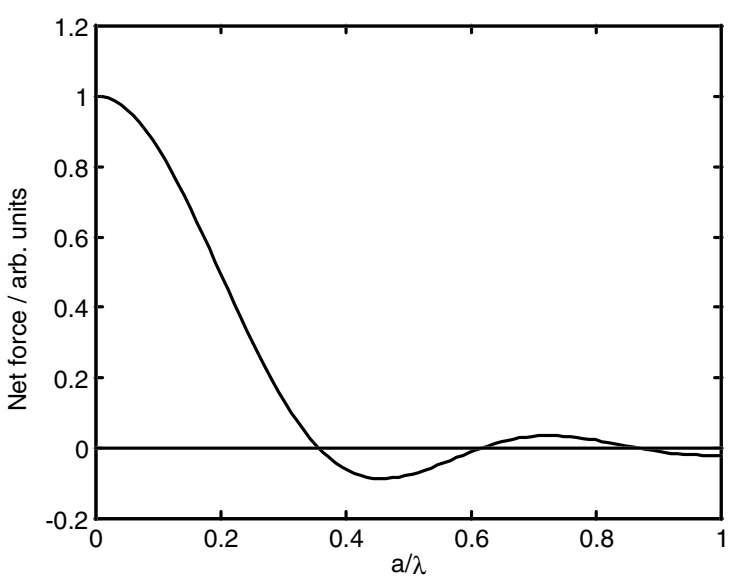

Figure 6. Decrease of net ponderomotive force with increasing particle size.

particles bunch under the influence of the ponderomotive forces in the bottom of the periodic potential wells. By considering spherical particles on axis that have radii of the same order as the radiation wavelength or less, it can be shown that the net force on such particles is zero when the particle radius satisfies the condition

$$
\frac{4 \pi a}{\lambda}=\tan \left(\frac{4 \pi a}{\lambda}\right) .
$$

In addition to the trivial solution $a=0,(21)$ is satisfied when $a / \lambda=0.36,0.62,0.86, \ldots$, as shown in figure 6 .

Particles with these sizes do not experience a net optical ponderomotive or gradient force and will not form part of any density grating. It can be envisaged that this could be used as the basis of novel methods for discriminating between particles of differing sizes in a sample. An illustrative example is that of a collection of particles flowing slowly in the $z$-direction, i.e. along the axis of the trap. Particles with sizes which did not satisfy (21) would be trapped, whereas those with sizes which satisfy (21) would pass through the standing-wave trap unhindered. The desired particle size to be 'filtered' could be changed by varying the radiation wavelength. A variation on this idea would be a stationary collection of particles and a moving standing wave trap, created by slightly detuning the light propagating in the $+z$-direction from that in the $-z$-direction. The moving standing-wave trap would drag or 'skim off' particles with sizes which did not satisfy (21), leaving behind only those for which equation (21) is satisfied.

\section{Discussion}

We have presented an outline of the theory of CRS and an example simulation of a collection of free latex microparticles pumped by a strong laser field. The results of a simple CRS model predict the spontaneous formation of an intense backscattered (probe) field and a strong particle density grating.

Results of preliminary experiments involving trapping of $\mathrm{TiO}_{2}$ particles in a standing-wave trap were also presented. These preliminary experiments have not been performed in a direct attempt to observe CRS (in the experiments there are two counterpropagating pump fields such that the ponderomotive forces do not have to spontaneously develop from the pump/probe interaction). However, they provide useful information about the number of particles which we can expect to participate in proof-of-principle experimental studies of CRS. In addition to the role of these experiments with regard to CRS, it was shown that they also represent a novel method of optical particle characterization.

The CRS simulation presented here showed the evolution to occur on a $\mu \mathrm{s}-\mathrm{ms}$ timescale. Great effort has been applied in the standing-wave trap experiments to maximize the time frame over which a collection of particles remain in the scattering volume. In the case when the thermophoretic force dominated the particle motion, the particle velocity was $\sim 3 \mathrm{~mm} \mathrm{~s}^{-1}$ which translates to a time of $20 \mathrm{~ms}$ to traverse a distance (roughly equal to the pump beam waist) of $60 \mu \mathrm{m}$. Simulations with an infra-red pump have indicated that this is not a sufficient time frame by an order of magnitude for CRS to build up. Therefore it is required that the steps outlined to minimize convection (lower medium gas pressure, more tightly focused pump beam) are taken.

CRS by nanoparticles at visible wavelengths would occur on a faster $\mu$ s timescale. At first, this relaxes the requirements of the pump intensity and medium pressure. However, the Brownian motion of these very small particles may be problematic in this regime. In a given time interval $t$, the mean Brownian displacement $x_{r m s}$ of a particle is given by

$$
x_{r m s}=\left[\frac{k_{B} T C_{c}}{3 \pi \eta a} t\right]^{1 / 2} .
$$

For example, for a latex particle $(a=50 \mathrm{~nm})$ in air at standard temperature and pressure, $x_{r m s} \sim 0.1 \mu \mathrm{m}$ in $10 \mu \mathrm{s}$. This is equivalent to $\lambda / 4$ at the optical wavelength which is half the distance between standing-wave anti-nodes, i.e. the distance such that the scattered radiation from this particle is exactly $\pi$ out of phase with that of bunched particles. With this Brownian motion acting to wash out any collective behaviour, it seems sensible to take the so described measures to minimise other undesired processes, namely medium convection.

\section{Acknowledgments}

We would like to thank the EPSRC and Royal Society of Edinburgh for supporting this work.

\section{References}

[1] Miles R B, Lempert W R and Forkey J N 2001 Laser Rayleigh scattering Meas. Sci. Technol. 12 R33-51

[2] McNeil B W J and Robb G R M 1998 Collective Rayleigh scattering from dielectric particles: a classical theory of the collective atomic recoil laser Opt. Commun. 148 54-8

[3] Smith P W, Maloney P J and Ashkin A 1982 Use of a liquid suspension of dielectric spheres as an artificial Kerr medium Opt. Lett. 7 347-9

[4] Moroz A 2000 Photonic crystals of coated metallic spheres Europhys. Lett. 50 466-72

[5] Cao H et al 1999 Random laser action in semiconductor powder Phys. Rev. Lett. 82 2278-81 
[6] Bonifacio R, Casagrande F, Cerchioni G, De Salvo Souza L, Pierini P and Piovella N 1990 Theory of the high-gain free-electron laser and superradiance Riv. Nuovo Cimento 13

[7] Bonifacio R, De Salvo L, Narducci L M and D'Angelo E J 1994 Exponential gain and self-bunching in a collective atomic recoil laser Phys. Rev. A 50 1716-24

[8] Ashkin A, Dziedzic J M, Bjorkholm J E and Chu S 1986 Observation of a single-beam gradient force optical trap for dielectric particles Opt. Lett. 11 288-90

[9] Ashkin A 1997 Optical trapping and manipulation of neutral particles using lasers Proc. Natl Acad. Sci. USA 94 4853-9

[10] Zemanek P, Jonas A, Sramek L and Liska M 1998 Optical trapping of Rayleigh particles using a Gaussian standing wave Opt. Commun. 151 273-85
[11] Harada Y and Asakura T 1996 Radiation forces on a dielectric sphere in the Rayleigh scattering regime Opt. Commun. 124 529-41

[12] Talbot L, Cheng R K, Schefer R W and Willis D R 1980 Thermophoresis of particles in a heated boundary layer J. Fluid Mech. 101 737-58

[13] Jaroszynski D A et al 2000 The Strathclyde terahertz to optical pulse source (TOPS) Nucl. Instrum. Methods Phys. Res. A $445317-19$

[14] Gouesbet G, Maheu B and Grehan G 1988 Light scattering from a sphere arbitrarily located in a Gaussian beam, using a Bromwich formulation J. Opt. Soc. Am. A 5 1427-43

[15] Kodas T T and Hampden-Smith M 1999 Aerosol Processing of Materials (New York: Wiley) 